\title{
Cooperation in Repeated Rock-Paper-Scissors Games in Uncertain Environments
}

\author{
Peter Andras ${ }^{1}$ \\ ${ }^{1}$ School of Computing and Mathematics, Keele University \\ p.andras@keele.ac.uk
}

\begin{abstract}
Cooperation among selfish individuals provides the fundamentals for social organization among animals and humans. Cooperation games capture this behavior at an abstract level and provide the tools for the analysis of the evolution of cooperation. Here we use the Rock-Paper-Scissors (RPS) game with positive and negative draw outcomes to study the evolution of cooperative behavior in communities of simulated selfish agents. The agents communicate to each other using a probabilistic language and the cooperation game is set in an uncertain resource generation context. The offspring of the agents may clump together or may spread out, simulating the easy and difficult identification of possible cooperation partners. The results show that more uncertainty leads to more cooperation both in positive and negative draw games. Surprisingly we found that in negative draw games the level of cooperation is statistically significantly higher, although close to, the level that would be expected from random choice of RPS decisions. We also analyzed language complexity correlates of cooperation. The agent-based simulations and the results described here are applicable to social institutions or ecological systems with more than two, non-transitively comparable, decision states that can be described abstractly as RPS games.
\end{abstract}

\section{Introduction}

Cooperation is very important in human society as it is at the foundation of all social institutions (Smaldino, 2018; Pletzer et al, 2018). In general, cooperation among selfish individuals is found in the context of many animal communities and even among plants (Moscovice et al, 2017; Callaway et al, 2002). Mechanisms that aim to explain the emergence and evolution of cooperation include classical and stochastic inclusive fitness leading to various forms of kin-selection (Kennedy et al, 2018), direct and indirect reciprocity (Rand and Nowak, 2013) and other approaches.

The majority of theoretical and experimental studies of cooperation use games with two outcomes, e.g. Prisoner's Dilemma game, as tools of abstract conceptualization of the interactions that may lead to cooperation decisions (Rand and Nowak, 2013). However, in real world scenarios often the case is that individuals participate in interactions that have multiple outcomes of varying utility for the involved participants (e.g. contracts between firms, rewarding/punishing employees, participation in rituals).

To model multiple outcome real life scenarios we can use multiple-outcome games. One such game is the Rock-PaperScissors (RPS) game with three outcomes, where 'rock' beats 'scissors', 'scissors' beat 'paper' and 'paper' beats 'rock', and the three draw options can be considered as equivalent of cooperation (of possibly three different kinds). There are many examples in the natural world, which follow the RPS rules. For example, several predator-prey systems with three or more species follow RPS (or extended RPS) dynamics (Edwards and Schreiber, 2010; Kerr et al, 2002), pricing and marketshare games between firms may follow RPS rules (Kovac and Schmidt, 2013; Hopkins and Seymour, 2002). Other examples that can be conceptualized using the RPS framework include volunteering in the context of provision of public goods (Semman et al, 2003), choice of mating behavior in lizards (Sinervo et al, 2007), and dynamics of species diversity (Reichenbach et al, 2008).

While in many real world realizations of RPS games the focus is on the dynamics of the three decision or behavior options, in some cases the draw options play the central role. For example, in pricing games, the draw options may represent stable prices (Cason et al, 2012) or in species interactions the draw options of self-restraint may represent the stable growth path for all participants (Nahum et al, 2011). In these cases the RPS game is played as a cooperation game, with the draws representing the cooperation decisions, e.g. self-restraint in growth or in pricing. An important difference between RPS games played as cooperation games and those which are played differently is that the outcome associated with the draw is sufficiently positive, i.e. beneficial for the participants, although not as much as the winning outcome. In other RPS games the outcome associated with the draw may be more negative than the outcome of losing a game. We note that in some natural cases of RPS game the draw is never an option, e.g. competition between different sub-species, where each one has only one behavioral option (Kirkup and Riley, 2004).

Here we use and agent-based simulation environment to explore the evolution of cooperation in the context of RPS games. The agents in our environment communicate their intentions and play an uncertain resource game, where the actual outcome of the game is drawn from a probability distribution over a range of possible outcomes. The agent's lifespan and number of offspring depend on the amount of resources that they accumulate. The asexually produced offspring inherit the communication language and inclination to cooperation decisions of the parent with minor random variations.

We explored games with positive draw outcome, where we expect high level of cooperation and also games with negative draw outcome, where we do not expect much cooperation (i.e. 
above the default level corresponding to random choice of agent decisions). We also investigated the impact of clustered and spread-out arrangement of the offspring of the agents, which represents the easy and difficult identification of likely cooperation partners (i.e. cooperation is more likely among the offspring of an agent, which had high inclination to make cooperative decisions).

The rest of the paper is structured as follows. First we review briefly the relevant literature. Next expand the discussion of cooperation in the context of RPS games. Next we describe the simulation environment in details. Then we present the results and discuss the implications of these. Finally the paper is closed with the conclusions.

\section{Background}

The RPS game originates in East Asia (China and Japan) and has been played for many centuries. RPS became popular in Europe in the 1920s (Schwab, 2015). Today it is one of the early strategy games that children learn in school and there are also championships organized for adult players. The game has been studied in the context of game theory and its applications, for example in biology, economics and social institutions (Cason et al, 2012; Semmann et al 2003).

There are several examples of RPS games in nature and in social context. In side-blotched lizards (Uta stansburiana) males may have three different variations of their throat color, orange, blue and yellow, each corresponding to a different mate choice strategy (i.e., aggressive defense of large territory, defense of small territory, sneaking through territories with a female-like look) (Sinervo and Lively, 1996; Sinervo et al, 2007). The three strategies form an RPS-like game, according to data from field experiments, orange is better than blue, yellow is better than orange and blue is better than yellow and the frequency of male lizards with these behavior features changes from year-to-year accordingly (Sinervo and Lively, 1996). Another example is the case of Escherichia coli bacteria, which may produce an antibacterial toxin (colicin) at some metabolic expense (variant $\mathrm{C}$ ), may have resistance to this toxin with somewhat less metabolic expense (variant R), and may be sensitive to the toxin, but grow quicker than the other two versions (variant S) (Kirkup and Riley, 2004). The S variant grows quicker than the $R$ variant ( $S$ wins against $R$ ), the $R$ variant grows quicker than the $C$ variant ( $R$ wins against $\mathrm{C}$ ), and the $\mathrm{C}$ variant kills the $\mathrm{S}$ variant (C wins against $\mathrm{S}$ ) (Kirkup and Riley, 2004). In the context of product pricing with well informed and not well informed buyers, a medium price may beat a high price and a low price may beat a medium price, but a high price may beat the low price (i.e., the price may switch to become a quality indicator) (Cason et al, 2012; Hopkins and Seymour, 2002).

RPS games have been analyzed mathematically to reveal key strategies (Sandholm et al, 2008). One such strategy is the replicator dynamics, when agents replicate the choice of the last opponent. Another strategy is the projection strategy, when an agent changes its choice depending on the inverse of the popularity of its own latest decision choice (Sandholm et al, 2008). RPS games have been analyzed in many formal settings, e.g. on networks (Alesina and Levine, 2011), with spatial constraints (Reichenbach et al, 2008), with extended state set (Peltomaki and Alava, 2008).

Cooperation may emerge in RPS games if the draw options have sufficiently positive outcomes (Nahum et al, 2011; Reichenbach et al, 2008). In such cases playing a draw leads to less benefit than winning a game, but also to more benefit than losing a game and the joint benefit of both partners is larger in the case of the draw than in the case of a winner - loser combination. For example, in the case of growth competition between behavioral variants of a species, the draw may allow on balance quicker growth for the whole population than the case when some are winning while others are losing through the interaction games (Nahum et al, 2011; Reichenbach et al, 2008). In the context of social games, restraint may bring more benefits at the community or institutional level then the playing of the game in winner - loser manner (Nahum et al, 2011).

RPS games have been studied extensively through modeling and simulations (Cliff and Miller, 1995; Schreiber and Killingback, 2012; Lubachevsky and Kanemoto, 2010). Most of these studies focus on the cyclical behavior of the agent community through playing the RPS game with alternating behavior/decision choices becoming dominant. In terms of analysis of the emergence of cooperation the computational studies focus on the role of spatial constraints in promoting restraint through clustering of individuals playing each decision/behavior option (Reichenbach et al, 2008).

Recently there have been several studies reporting on experimental RPS game playing with human players (Batzilis et al, 2014; Semmann et al, 2003). These investigated mainly RPS games in economic and social setting (e.g. pricing games, public goods games) documenting how real humans play in more realistic and non-abstract settings (Batzilis et al, 2014; Semmann et al, 2003).

Studies aimed to uncover the mechanisms that underlie cooperation among selfish individuals have considered the role of uncertainty (Andras et al, 2003; Andras, 2016). It has been shown that in the context of Prisoner's Dilemma type games more uncertainty leads to higher level of cooperation, but this has not been studied yet in the context of cooperation in RPS games. Natural world examples confirm that higher level of environmental uncertainty leads to more cooperative behavior among animals and plants (Callaway et al, 2002; Moscovice et al, 2017).

The ease of identification of prospective cooperation partners contributes also to the setting of the level of cooperation (Andras, 2016). It has been shown through agentbased simulations that more clustering of cooperators increases the level of cooperation, where clustering of cooperator is a way of representing the ease of identification of cooperators. Studies of cooperation in RPS games also have shown that spatial segregation of individuals with similar decision preferences favors the increase of the level of cooperation (Nahum et al, 2011; Reichenbach et al, 2008).

\section{Cooperation in the Rock-Paper-Scissors Game}

We noted above that RPS games can be used to study the evolution of cooperation if the draw outcomes are positive and possible. This setting aims to replicate cases of natural and social situations where the decisions or behavior that may be 
chosen by the actors are such that the draw is a possibility, e.g. spatial segregation of species (Nahum et al, 2011; Reichenbach et al, 2008), price choices of firms (Kovac and Schmidt, 2013). The RPS game is formulated using a pay-off matrix as shown in Table 1:

Table 1. Pay-off matrix of an RPS game

\begin{tabular}{|c|c|c|c|c|}
\hline 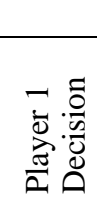 & \multicolumn{4}{|c|}{ Player 2 Decision } \\
\hline \multirow{4}{*}{ 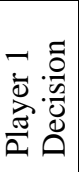 } & & $\mathrm{R}$ & $\mathrm{P}$ & $S$ \\
\hline & $\mathrm{R}$ & $(a, a)$ & $(d, e)$ & $(f, g)$ \\
\hline & $\mathrm{P}$ & $(e, d)$ & $(b, b)$ & $(h, k)$ \\
\hline & $\mathrm{S}$ & $(g, f)$ & $(k, h)$ & $(c, c)$ \\
\hline
\end{tabular}

such that $e>d, f>g, k>h, e>a, f>a, k>a, e>b, f>b, k$ $>b, e>c, f>c, k>c$. In order to specify an RPS with incentive for cooperation we also need to have $a>d, a>g, a$ $>h, b>d, b>g, b>h, c>d, c>g, c>h$ and that $2 a>$ $d+e, 2 a>f+g, 2 a>h+k, 2 b>d+e, 2 b>f+g, 2 b>h+k, 2 c$ $>d+e, 2 c>f+g, 2 c>h+k-$ this is called positive outcome for draws or positive draw outcome RPS. The settings can also be turned around to favor the avoidance of draws if $a<d$, $a<$ $g, a<h, b<d, b<g, b<h, c<d, c<g, c<h-$ this is called negative outcome for draws or negative draw outcome RPS. In general we do not have further constraints. However, to simplify the game, we may assume that $a=b=c, e=f=k, d$ $=g=h$, the corresponding pay-of matrix is shown in Table 2 .

Table 2. Pay-off matrix of a simplified RPS game

\begin{tabular}{|c|c|c|c|c|}
\hline & \multicolumn{4}{|c|}{ Player 2 Decision } \\
\hline \multirow{4}{*}{ 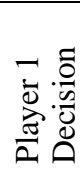 } & & $\mathrm{R}$ & $\mathrm{P}$ & $S$ \\
\hline & $\mathrm{R}$ & $(a, a)$ & $(d, e)$ & $(e, d)$ \\
\hline & $\mathrm{P}$ & $(e, d)$ & $(a, a)$ & $(d, e)$ \\
\hline & $\mathrm{S}$ & $(d, e)$ & $(e, d)$ & $(a, a)$ \\
\hline
\end{tabular}

In such case positive draw outcome is satisfied if $e>a>d, 2 a$ $>e+d$ and negative draw outcome is satisfied if $e>d>a$.

In the social context, cooperation games can be considered as abstract conceptualization of social institutions (Goist and Kern, 2018; Kube et al, 2014). RPS games can be seen as abstract representation of social games with multiple decision options, which are non-transitively comparable, i.e. if option 1 is better than option 2 and option 2 is better than option 3 then it does not follow that option 1 is better than option 3 , but rather the opposite of the latter applies. There are many examples of such settings in social institutions, for example, political parties may offer non-transitively comparable solution options to currently high priority socio-economic problems; different parts (e.g. directorates) of an organization may push for different priorities, which are non-transitively comparable, in terms of growth and investment focus; companies may try to sell non-transitively comparable goods or services to their potential clients, emphasizing different advantages of their products. Understanding how RPS games support the evolution of cooperative behavior can help the understanding, design and management of such social institutions.

In general we expect that in a positive draw RPS game that is played repeatedly the benefits of the draw option may attract actors towards playing this option due to the assumed drivers of cooperation (i.e. kinship, direct or indirect reciprocity, environmental uncertainty) (Rand and Nowak, 2013). However, there is always a temptation to play the decision option that trumps the recently played option and there is always a risk that the next game partner will play the trumping decision option. One possibility is the spatial segregation and low mobility of actors, such that segregated actors stick to the decision option that they play and do not succumb to the temptation of playing a winning option (Schreiber and Killinback, 2012; Reichenbach et al., 2008). However, this may not be practical in social institutions. An alternative may be the maintenance of a social compromise for a period, followed by a relatively quick shift to alternate social compromise. To some extent the validity of this process is supported by the evidence of periodic shifts in government or investment and growth focus in companies. Experimental investigation, for example, through computational simulations can help elucidate factors that drive and influence the maintenance of such processes.

In certain social situations a losing option gives more benefit to the actor choosing this option than the choice of a decision option that would lead to a draw. Ending up as loser in an interaction may trigger a compensation benefit, which might be psychological (e.g. learning a lesson from a loss), economic (e.g. compensation payment or investment) or social (e.g. providing a compensatory mean of influence in the institutional decision making processes). At the same time ending with draw as a result of the interaction, involves the same cost of emotional, economic or social investment as the loss, but will not trigger the compensatory benefit. An example is when political parties negotiate by deciding to not join the forming of a government, offering support for or forming a minority or low majority government and forming a grand coalition government. The three options may trump another in a nontransitive manner, while at the same time the matching options may bring less benefit to the parties than the option where they are on the losing end, due to different kinds of benefit (e.g. government posts and ability to criticize more or less freely the government in place). State provided subventions and economic interventions are often forms of compensations for losing firms in the context of competitive economies, while the ability to carry on the competition between firms in an undecided draw does not trigger the willingness of the state to intervene and provide compensation for the loser. Setting a minimum wage by the state can also be seen as a compensation for a loss or perhaps rather a prevention of a drawn out playing of a draw between employers and employees. Here the draw happens by employees holding out without taking low paid jobs and employers holding out by not offering higher pay, leading to high unemployment and low level of production. Preventing this draw, by rewarding the loser through the imposition of a minimum wage, the employees are kind-of bribed into accepting lower than preferred wages in combination with a wage floor and employers are made to accept a cut in their profit and a reduction of their production potential. In some countries political organizations of minorities may gain some level of representation if they clearly lose their electoral battles, but may not qualify for this is they gain sufficient number of votes and are rather seen as achieving a regular draw in the political arena against other non-minority political organizations. Similarly state provided funding arrangements for such organizations may provide more support to those who are clearly on the losing end in open competition. 
In the case of negative draw RPS games we expect that in all conditions there is no increase in the level of draws compared to what is the result of random effects on the decision choices. However, it is still interesting to see if this expectation is confirmed by computational simulations and also to see if any factor in the setting of negative draw RPS games may influence the level of accidental cooperation in these games.

In our computational simulation study we analyze the role of environmental uncertainty and ease of identification of potential cooperators (implemented through agent offspring mobility: clustered offspring representing easy identification of potential cooperators; spread-out offspring representing the difficult identification of potential cooperators) on the level of cooperation in the context of positive and negative draw RPS games. Both factors have been shown to influence the level of cooperation in Prisoner's Dilemma games, so it is expected that they have an influence in the context of RPS games as well (Andras, 2016).

In addition to the level of cooperation we also aim to use language complexity correlates of cooperation (Andras, 2008; Andras, 2016) to analyze the impact of uncertainty and ease of cooperator identification on the level of cooperation in RPS games. The language complexity metrics that we use are the average length of communications and the average variability of the use of language rules among the agents. In general we expect that in the case of positive draw RPS games, where cooperation is preferred, the language complexity metrics will decrease with increasing uncertainty of the environment. In the case of negative draw RPS games we do not expect a systemic variation of the language complexity metrics with environmental uncertainty of variation of the ease of identification of potential cooperators.

\section{Rock-Paper-Scissors Simulation Environment}

Our agent-based simulation environment works in a torus-like flat space (i.e. the opposite edges of a rectangle are joined together). The agents move in this environment randomly following a Brownian motion. The same spatial position may be occupied by multiple agents.

The agents communicate with each other about their intentions. In each time turn, each agent tries to pick a partner from its neighborhood of agents. Agents that find a partner play an uncertain resource generation game with their partner such that they reach their game decisions through their communications. If the communications last too long (too many symbols are produced before reaching the decision) the agents disengage.

The language of the agents is defined using a set of probabilistic production rules

$$
R:\left(\omega_{\text {own }}, \omega_{\text {other }}\right) \rightarrow\left(\omega_{\text {new }, 1} ; p_{1}\right), \ldots,\left(\omega_{\text {new }, k} ; p_{k}\right)
$$

where $\omega_{\text {own }}$ and $\omega_{\text {other }}$ are the last communication symbols produced by the agent (own) and its partner (other), and $\omega_{\text {new, } i}$ and $p_{i}$ are the i-th communication symbol, which may be produced next and the probability of the production of this symbol - the probabilities $p_{i}$ sum up to 1 . The symbol set is common across all agents and includes the following symbols with the corresponding meaning ' 0 ' - wait, ' $s$ ' - start meaningful communication, 'i' - continue communicating, ' $y$ '
- ready for decision making, ' $n$ ' - does not want to communicate, 'h' - 'rock', 't' - 'paper', and 'r' - 'scissors'. Each agent has a willingness (probability) to produce as their decision 'rock', 'paper' or 'scissors' following the reaching of the state, when both agents communicated the symbol ' $y$ ' to each other. The probabilities for the production rules are specific for each individual agent. This agent language builds on earlier work used for simulation of Prisoner's Dilemma games and further details about it can be found in earlier papers (Andras, 2003; Andras, 2008).

The uncertain resource game is played by the agents according to a pay-off matrix like the one shown in Table 2. However in this case the pay-offs are uncertain and they are sampled from uniform distributions for which the mean value is given by the pay-off matrix and the half-width of the distribution (corresponding to the standard deviation) is given by the uncertainty of the environment, $\sigma$, multiplied by the length of the communications that took place between the agents participating in the game until they reached their decision. The mean values in the pay-off matrix depend on the amount of resources the agents. Higher uncertainty $\sigma$ and longer decision reaching communications imply larger halfwidth for the distribution, which means higher chance to get much smaller or much larger values than the mean as the payoff of the actual game played by the agents.

We had two kinds of settings for the pay-off matrix: positive draw RPS and negative draw RPS, in order to be able to investigate both kinds of RPS games. The function that determines the mean values for the pay-off matrix is set such that the values in the pay-off matrix always satisfy the inequalities required for the two kinds of RPS games (the setting is different for the two kinds of RPS games).

The agents live for 60 time turns at most - they may die earlier if they run out of resources (existence in each time turn costs a fixed amount of resources). At the end of their life, if they have at least the average amount of resources, considering the current agent population, the agents produce offspring asexually. The offspring share equally their parent's resources and their number depends on the available resources of the parent agent according to the following equation

$$
N_{\text {new }}=\left[\alpha \cdot\left(\left(\rho-\rho_{m}\right) / \rho_{s}\right)+\beta\right]
$$

where $N_{\text {new }}$ is the number of the offspring, $\rho, \rho_{m}, \rho_{s}$ are the amount of resources of the parent agent, the mean amount of resources and the standard deviation of the resource amounts in the current agent population, and $\alpha$ and $\beta$ are parameters. The offspring inherit the language of their parent, including their willingness to produce the decisions 'rock', 'paper' and 'scissors', with minor changes of the probabilities of the language production rules.

The offspring may start in a clustered manner form the location of the parent agent or may be dispersed randomly throughout the whole space of the simulation. The clustered starting setting of the offspring corresponds to the easy identification of prospective cooperator agents, i.e. the offspring of an agent have similar preferences for producing the 'rock', 'paper', 'scissors' decisions, so being clustered they are likely to play a draw - cooperate - if any of the three decisions is more preferred by them than the other two decisions, which is usually the case. The spread-out, dispersed, offspring represents the difficult identification of prospective cooperators, since the offspring are not clustered and it is not 

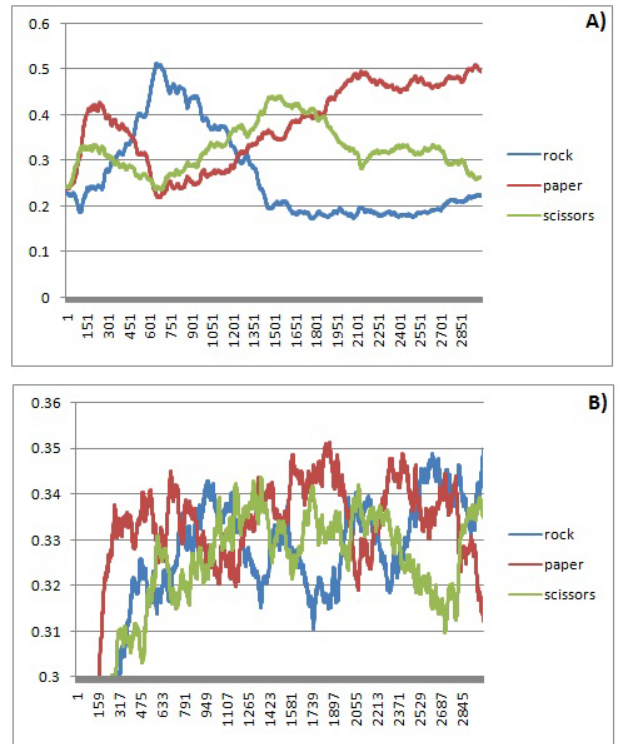

Figure 1. Typical variation of the three decision choices ('rock', 'paper', 'scissors') for two simulated agent worlds: A) positive draw RPS with clustered offspring; B) negative draw RPS with spread-out offspring. The horizontal axes show time, the vertical axes show the frequency of the decision choices.

predictable the decision making preference of the neighboring agents.

The agent world evolves through time turns. In each time turn the agents pick partners, communicate and play the resource game. The agents that reach the end of their life produce their offspring if they have sufficient resources. As agents inherit their parent's language and decision preferences with minor random changes, the evolution may lead to the emergence of a stable pattern of the level of cooperation.

We measured the level of cooperation as the percentage of agents reaching a draw decision, within the current agent population. We also measured correlates of cooperation: the average length of agent communications as the average number of symbols produced by the agent until they reached their decision in the resource game; and the language variability measured as the average standard deviation of the distributions of the probability values associated with language production rules across the current population of the agents.

\section{Results and Discussion}

The simulated agent worlds ran for 3000 time turns. Each simulated world started with around 1800 agents with randomly set language probabilities. We considered three levels of environmental uncertainty $(\sigma=0.3,0.5$ and 0.7$)$ Each simulation setting was run 20 times and we calculated the average level of cooperation, communication length and language variability for each time turn. We analyzed these averaged data. The standard deviations of the data are small compared to the averages and these are not shown in the figures to avoid cluttering.

We also measured the frequency of 'rock', 'paper' and 'scissors' decisions to check whether these alternate across the

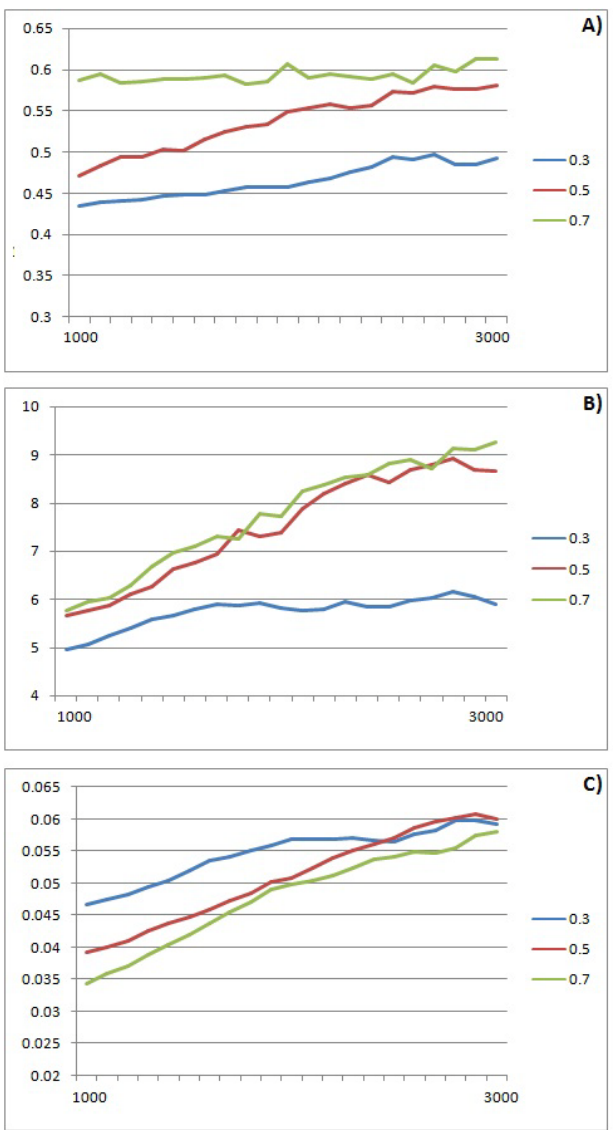

Figure 2. Evolution of the level of cooperation (A), average communication length (B) and language variability (C) during the last 2000 time turns of the simulations with positive draw RPS games with clustered offspring. The horizontal axes show the time and the vertical axes show the measured metrics. The uncertainty levels are indicated by the line colors blue -0.3 , red -0.5 , green -0.7 .

simulations as expected from the RPS game. Figure 1 shows two typical cases, one for the positive draw RPS with clustered offspring (A) and the other for negative draw RPS with spread-out offspring (B). In both cases, initially some of the agents do not produce any of these decisions as they do not reach the decision sufficiently quickly (at this stage around a quarter of the agents produces each of three decision options and around a quarter of the agents do not reach any of these decisions). The data in Figure 1 shows that indeed, the three decisions alternate in their frequency. In the case of positive draw RPS with clustered offspring the alternation is much slower than in the case of the negative draw RPS with spreadout offspring. We also note that in the positive draw RPS the dominant decision choice is much more frequent than in the negative draw RPS, which is in line with the draws being beneficial in the former and not so in the latter.

The evolution of cooperation and the communication length and language complexity metrics for positive draw RPS games with clustered offspring are shown in Figure 2. The results show that similar to results about Prisoner's Dilemma games in uncertain environments, we find that more uncertain environments imply higher level of cooperation. The results 


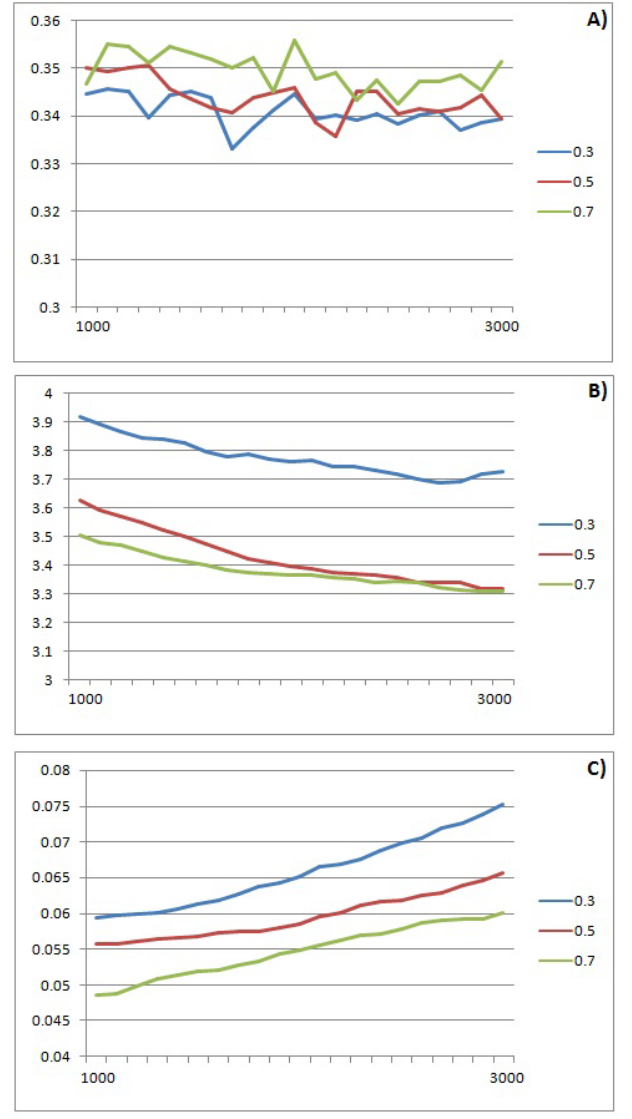

Figure 3. Evolution of the level of cooperation (A), average communication length (B) and language variability (C) during the last 2000 time turns of the simulations with negative draw RPS games with clustered offspring. The horizontal axes show the time and the vertical axes show the measured metrics. The uncertainty levels are indicated by the line colors blue -0.3 , red -0.5 , green -0.7 .

also show that the more uncertainty is consistent with longer communications and with lower variability of the language rule probabilities among the agents. We also note the upward trends of the language complexity metrics over time.

In the case of simulations with negative draw RPS games and clustered offspring we found that the differences are small between the levels of cooperation (all a bit over 0.33 , which is the expectation for random choice of the three decision options) for the different levels of uncertainty, but these differences are statistically significant (the difference between the average level of cooperation for the last 2000 time turns for the highest and lowest level of uncertainty is 0.0095, which is shown by the t-test to be statistically very significant, $\mathrm{p}$-value = 0 ). Again we found that the language variability is lower for higher environmental uncertainty for these games and for all levels of uncertainty there is growth trend over time for this metric. We also found that for these games the average communication length is lower for more uncertain environments and for this metric the trend is further reduction. The results are shown in Figure 3.

For the case of positive draw RPS games with spread-out offspring the results are shown in Figure 4. We found that the
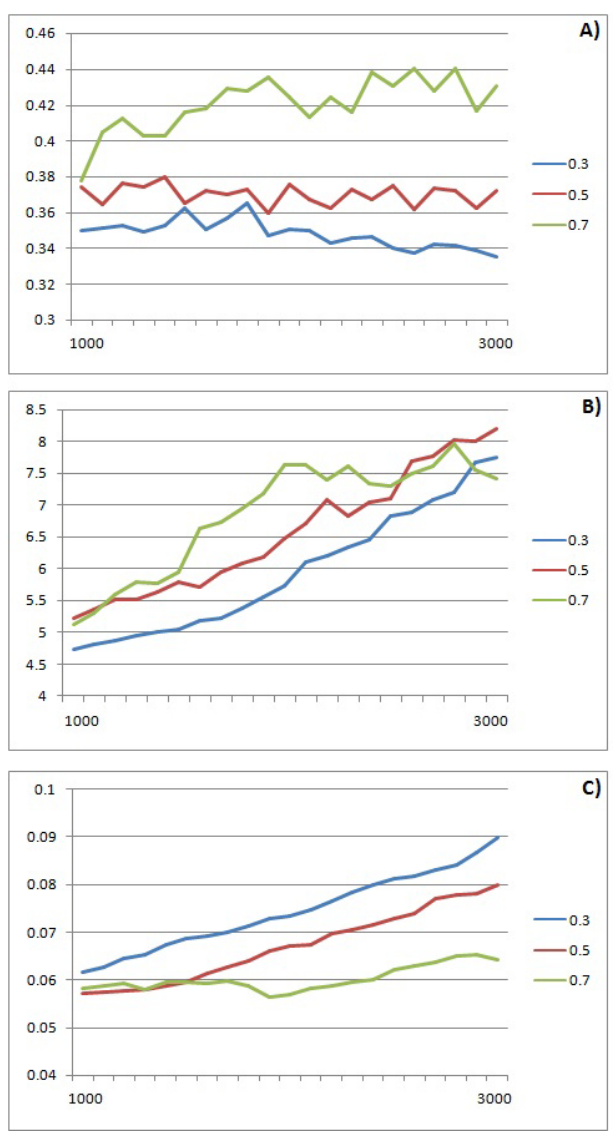

Figure 4. Evolution of the level of cooperation (A), average communication length (B) and language variability (C) during the last 2000 time turns of the simulations with positive draw RPS games with spread-out offspring. The horizontal axes show the time and the vertical axes show the measured metrics. The uncertainty levels are indicated by the line colors blue -0.3 , red -0.5 , green -0.7 .

level of cooperation increases with the level of uncertainty, although to less extent than in the case of clustered offspring (this is as expected). The results show that the average communication length is longer and the language variability is smaller in more uncertain environments, both also having an upward trend over time. The average communication lengths are smaller than the corresponding lengths for simulations with clustered offspring and the corresponding language variability measures are larger than in the case of clustered offspring.

Finally, in the case of negative draw RPS games with spread-out offspring we found that the levels of cooperation are similar and close to 0.33 (the expectation for random choice of the decisions), but the averages over the last 2000 time turns are still statistically significantly different for different uncertainty levels (the matching difference noted previously is 0.0024 , which is statistically very significant according to the t-test with $\mathrm{p}$-value $=3 \times 10^{-123}$ ). The language metrics behave in a similar manner like in the case of negative draw RPS games with clustered offspring, the only difference being that in this case the values are larger both for the average communication length and the language variability. The results are displayed in Figure 5. 

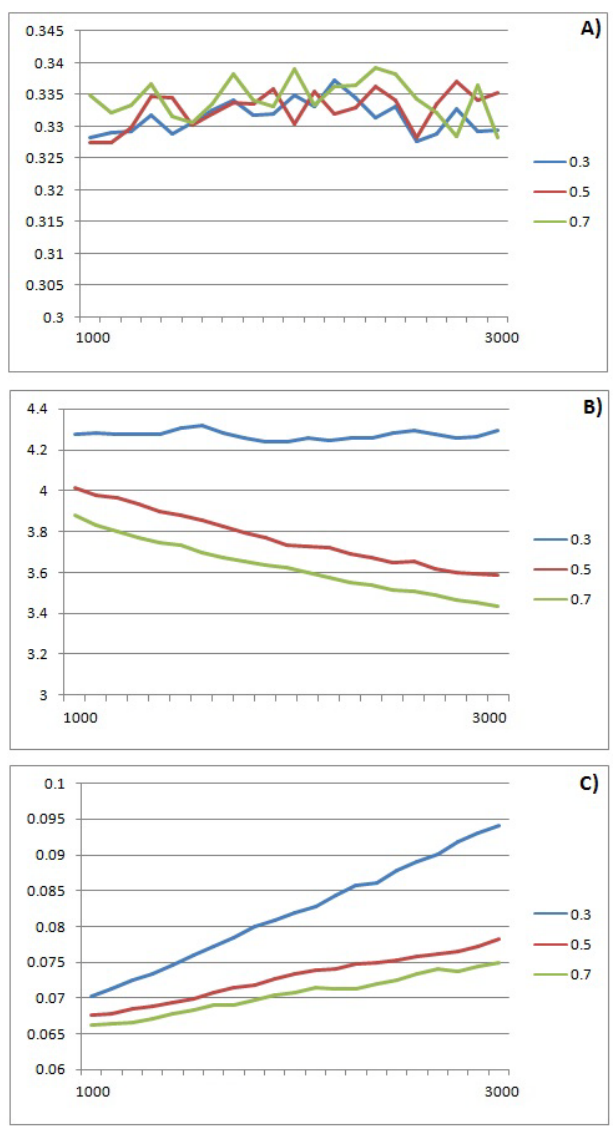

Figure 5. Evolution of the level of cooperation (A), average communication length (B) and language variability (C) during the last 2000 time turns of the simulations with negative draw RPS games with spread-out offspring. The horizontal axes show the time and the vertical axes show the measured metrics. The uncertainty levels are indicated by the line colors blue -0.3 , red -0.5 , green -0.7 .

Our results show that the language variability follows the same ordering by uncertainty and the same trend over time for all considered cases, irrespective of whether the RPS game is with positive or negative draw, or whether the offspring of the agents are clustered or spread-out. This suggests that lower communication uncertainty combines with higher environmental uncertainty to reduce the combined objective uncertainty. This is further reduced in terms of experienced uncertainty by higher level of cooperation associated with higher level of environmental uncertainty (Andras et al, 2006).

We also found more language variability for simulations with spread-out offspring than for those with clustered offspring. This is expected given the more mixing of the agents with different versions of the language in the former case compared with the latter one. This is also expected in natural RPS games where the identification of potential cooperators / non-cooperators is more difficult and consequently the game playing language is more diverse than in cases where this identification is easier.

The ordering of the length of communications graphs gets reversed between positive and negative draw RPS games and the trend over time gets also reversed (upward for positive draw and downward for negative draw RPS). This shows that this aspect of language complexity relates more to the nature of the games (positive vs. negative / preferred vs. un-preferred draw). When draws are preferred it takes longer to reach the game decisions, while when draws are un-preferred, game decisions are reached more rapidly. Comparing these graphs between the cases of spread-out and clustered offspring simulations we find that the communications take longer in the former case for negative draw RPS games, but the situation is less clear cut in the case of positive draw RPS games.

The reverse ordering of the communication length graphs compared to the ordering of the language variability graphs, in the case of positive draw RPS games is puzzling. This ordering indicates that reaching the game decisions takes longer, making the outcomes more uncertain, as the environmental uncertainty grows. The previously discussed uncertainty considerations would suggest that the opposite should happen, as it is in the case of negative draw RPS games. This finding suggests that possibly the benefits of cooperation in reduction of the experienced uncertainty outweigh the negative impact of increased uncertainty due to the longer time that it takes to reach the game decisions. Given that cooperation is less beneficial in negative draw RPS games, we do not see this effect in the case of these games.

As an example of the positive draw RPS game, we may consider a version of coalition decision making in politics, with offering coalition, demanding more gains and breaking the coalition being the three options on both sides and positive outcomes associated with the draw situations. Our results discussed above suggest that in such cases, the range of topics that are not easily agreed gets reduced as the economic-socialpolitical environment gets more uncertain. At the same time, the length of coalition negotiation gets extended as the environment gets more uncertain (Martin and Vanberg, 2003).

As expected, we find less cooperation for the case of agent societies with spread-out offspring than for those with clustered offspring. Interestingly, we found that there is significantly more cooperation in more uncertain environments even for negative draw RPS games (i.e. when cooperation representing draws are un-preferred). For the case of spreadout offspring with negative draw RPS the cooperation levels get very close to the level expected for random choice of game decisions, but still they are statistically significantly above this. This is a puzzling result, which perhaps indicates that the uncertainty reduction effect of cooperation is beneficial even if cooperation in itself leads to relative losses. These losses are relatively certain compared to the more uncertain gains. This suggests that in natural social realizations of RPS games with negative draws we can still expect some level of intentional cooperation above what can be expected purely by chance.

\section{Conclusions}

In this paper we report about computational experiments exploring the evolution of cooperation in the context of RPS games, where cooperation is interpreted as the draws in the game. We used an agent based simulation, with communicating agents playing a resource generation game in an uncertain environment, with or without dispersion of the offspring of the agents. The results show that higher 
environmental uncertainty is associated with higher level of cooperation in all cases, including those when draws imply a negative outcome in the RPS games. We also found that spreading-out of the offspring (an implementation of the difficult identification of potential cooperators) reduces the level of cooperation. In terms of language complexity correlates of cooperation we found that the language variability decreases as environmental uncertainty increases, but the average communication length follows this pattern only in the case of negative draw RPS games. In the case of positive draw RPS the results suggests that the benefits of cooperation outweigh the uncertainty increasing effect of lengthier communications.

The results reported here are interesting because they allow the computational analysis of social institutions and biological interaction systems (e.g. animal communities, multi-species bio-systems), that can be conceptualized abstractly as RPS games where cooperation is implemented as draws. This goes beyond the usual computational models of cooperation that use Prisoner's Dilemma or similar two option games and expand the range social and biological systems that can be studied from the perspective of the emergence, evolution and maintenance of cooperation. In particular in the case of social institutions RPS games (and possible further extensions of them) can capture multi-choice decision making with nontransitive comparability of the decision options, which characterizes many real world social institutions.

Future work will look at the use of agent-based models of RPS games with cooperation to study the formation and behavior of social institutions. In this setting, we will look at the role of social learning (Andras, 2016) in RPS games and the use of this combination to capture more fully the behavior of social institutions.

\section{References}

Alesina, S. and Levine, J.M. (2011). A competitive network theory of species diversity. PNAS, 108: 5638-5642.

Andras, P. (2008). Uncertainty and communication complexity in iterated cooperation games. In: Proceedings of the Artificial Life XI Conference, MIT Press, pp.9-16.

Andras, P. (2016). Social learning, environmental adversity and the evolution of cooperation. In: Proceedings of the Artificial Life XV Conference, MIT Press, pp.290-297.

Andras, P., Roberts, G., and Lazarus, J. (2003). Environmental risk, cooperation and communication complexity. In Alonso, E., Kudenko, D., and Kazakov, D., editors, Adaptive Agents and Multi-Agent Systems, pp. 49-65. Springer-Verlag, Berlin.

Andras, P., Lazarus, J., Roberts, G., and Lynden, S. J. (2006). Uncertainty and cooperation: Analytical results and a simulated agent society. JASSS - Journal of Artificial Societies and Social Simulation, 9:1/7.

Batzilis, D., Jaffe, S., Levitt, S., List, J.A., Picel, J. (2014). How Facebook Can Deepen our understanding of Behavior in Strategic Settings: Evidence from a Million Rock-Paper-Scissors Games. Working Paper - Harvard University.

Callaway RM, Brooker, RW, Choler, P, Kikvidze, Z, Lortie, CJ, Michalet, R, Paolini, L, Pugnaire, FI, Newingham, B, Aschehoug, ET, Armas, C, Kikodze, D, \& Cook, BJ (2002). Positive interactions among alpine plants increase with stress. Nature, 417:844-847.

Cason, T.N., Friedman, D., Hokins, E. (2012). Cycles and instability in a Rock-Paper-Scissors population game: A continuous time experiment. Working Papers, UC Santa Cruz Economics Department, No.702.
Cliff D. and Miller G.F. (1995) Tracking the red queen: Measurements of adaptive progress in co-evolutionary simulations. In: Morán F., Moreno A., Merelo J.J., Chacón P., editors, Advances in Artificial Life, LNCS 929, Springer, Berlin.

Edwards, K.F. and Schreiber, S.J. (2010). Preemption of space can lead to intransitive coexistence of competitors. Oikos, 119: 1201-1209.

Goist, M. and Kern, F.G, (2018). Traditional institutions and social cooperation: Experimental evidence from the Buganda Kingdom. Research \& Politics, 5:1-9.

Hopkins, E. and Seymour, R.M. (2002). The stability of price dispersion under seller and consumer learning. International Economic Review, 43: 1157-1190.

Kennedy, P., Higginson, A.D., Radford, A.N., Sumner, S. (2018). Altruism in a volatile world. Nature, 555: 359-362.

Kerr, B., Riley, M.A., Feldman, M.W., Bohannan, B.J.M. (2002). Local dispersal promotes biodiversity in a real-life game of rock-paperscissors. Nature, 418: 171-174.

Kirkup, B.C. and Riley, M.A. (2004). Antibiotic-mediated antagonism leads to a bacterial game of rock-paper-scissors in vivo. Nature, 428: 412-414.

Kováč, E. and Schmidt, R.C. (2013). Market share dynamics in a duopoly model with word-of-mouth communication. Games and Economic Behavior, 83:178-206.

Kube, S., Schaube, S., Schildberg-Hörisch, H., Khachatryan, E. (2014). Institution formation and cooperation with heterogeneous agents. Institute for Labor Economics (Bonn) IZA Discussion Papers, No.8533.

Lubachevsky, I. and Kanemoto, S. (2010). Scale-free memory model for multiagent reinforcement learning. Mean field approximation and rock-paper-scissors dynamics. The European Physical Journal B, 76: 69-85.

Martin, L.W. and Vanberg, G. (2003). Wasting Time? The Impact of Ideology and Size on Delay in Coalition Formation. British Journal of Political Science, 33: 323-344.

Moscovice, L.R., Douglas, P.H., Martinez-Inigo, L., Surbeck, M., Vigilant, L., Hohmann, G. (2017). Stable and fluctuating social preferences and implications for cooperation among female bonobos at LuiKotale, Salonga National Park, DRC. American Journal of Physical Anthropology, 163:158-172.

Nahum, J.R., Harding, B.N., Kerr, B. (2011). Evolution of restraint in a structured rock-paper-scissors community. PNAS, 108: 1083110838.

Peltomäki, M. and Alava, M. (2008). Three- and four-state rock-paperscissors games with diffusion. Physical Review E, 78: 031906.

Pletzer, J.L., Balliet, D., Joireman, J., Kuhlman, D.M., Voelpel, S.C., Van Lange, P.A.M. (2018). Social value orientation, expectations, and cooperation in social dilemmas: A meta-analysis. European Journal of Personality, 32:62-83.

Rand, D.G. and Nowak, M. (2013). Human cooperation. Trends in Cognitive Sciences, 17:413-425.

Reichenbach, T., Mobilia, M., Frey, E. (2008). Mobility promotes and jeopardizes biodiversity in rock-paper-scissors games. Nature, 448: 1046-1049.

Sandholm, W.H., Dokumci, E., Lahkar, R. (2008). The projection dynamic and the replicator dynamic. Games and Economic Behavior, 64: 666-683.

Schreiber, S.J. and Killingback, T.P. (2012). Spatial heterogeneity promotes coexistence of rock-paper-scissor metacommunities. Theoretical Population Biology, 86: 1-11.

Schwab, K. (2015). A Cultural History of Rock-Paper-Scissors. The Atlantic, December 23, 2015.

Semmann, D., Krambeck, H.-J., Milinski, M. (2003). Volunteering leads to rock-paper- scissors dynamics in a public goods game. Nature, 425: 390-393.

Sinervo, B. and Lively, C.M. (1996). The rock-paper-scissors game and the evolution of alternative male strategies. Nature, 380: 240-243.

Sinervo, B., Heulin, B., Surget-Groba, Y., Clobert, J., Miles, D.B., Corl, A., Chaine, A., Davis, A. (2007). Models of Density-Dependent Genic Selection and a New Rock-Paper-Scissors Social System. The American Naturalist, 170: 663-680.

Smaldino, P.E. (2018). Social identity and cooperation in cultural evolution. To appear in the Behavioural Processes. 